\title{
Loss and gyroscopic sensing using Rayleigh scattering in a fiber ring resonator
}

\author{
A. Küng , L. Thévenaz and Ph. A. Robert \\ Swiss Federal Institute of Technology (EPFL), Laboratory of Metrology, \\ CH - 1015 Lausanne, Switzerland \\ Tel: +41216934809 Fax: +41216932614 E-Mail: Alain.Kueng@epfl.ch
}

\begin{abstract}
Rayleigh backscattering measurements on a fiber ring resonator enables to monitor loss and gyroscopic motion experienced by the ring. This easy-to-use technique yields to very sensitive fiber loss sensors and polarization-insensitive and low-cost gyroscopes.
\end{abstract}

The idea of interrogating a fiber-optic ring using an optical time-domain reflectometer (OTDR) was originally developed for checking the linearity of the OTDR. But it tuns out that the trace delivered by the OTDR is strongly modified when the quality factor of the ring resonator increases. Thus a complete model of the OTDR response taking account on the coherence properties of the Rayleigh

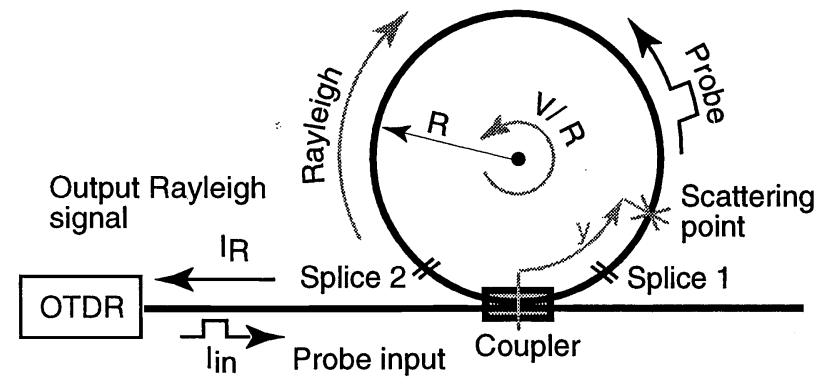

Fig. 1. Schematic description of a Rayleigh fiber ring gyroscope of radius $\mathrm{R}$, rotating at angular speed $v / R$.

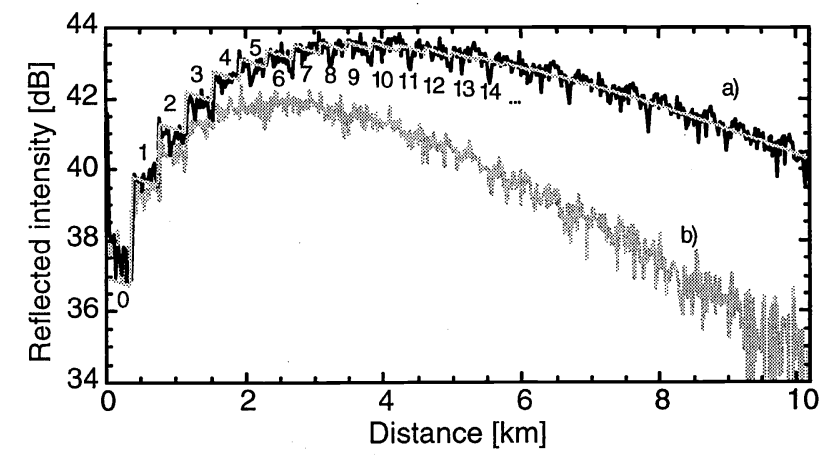

Fig. 2. OTDR trace of a $765 \mathrm{~m}$ long fiber ring resonator at rest with a coupler coefficient $k=95 \%$ and numerical fit of Eq. (6). The first step orders are indicated. The calculated cavity feedback coefficient is: a) $k r=89.5 \%$, b) $k r=79.7 \%$ due to an additional fiber bending loss diffusion is given in this paper. We will show that the relevant ring parameters (loss and coupling coefficient) can be extracted by simply measuring the position of the maximum of the OTDR trace ${ }^{1}$ making this technique very sensitive for characterizing fiber ring resonators or for designing sensors based on fiber loss modification. We will also show that this configuration is sensitive to the Sagnac effect and yields to a polarization-insensitive and low-cost gyroscope.

\section{PRINCIPLE AND MODELING}

The ring structure has the unique feature to let the OTDR pulse circulate many times in the ring, in the counterclockwise direction as described in Fig. 1, while generating Rayleigh backscattering. The backscattered light also circulates in the ring but in the clockwise direction and part of it is directed onto the OTDR detection stage at each turn. This re-circulation of the OTDR pulse combined together with the recirculation of the Rayleigh backscattered light allows different Rayleigh contributions to be detected simultaneously resulting in a step-like OTDR trace as shown in Fig. 2. The first steps are given by few contributions of great intensity. As 


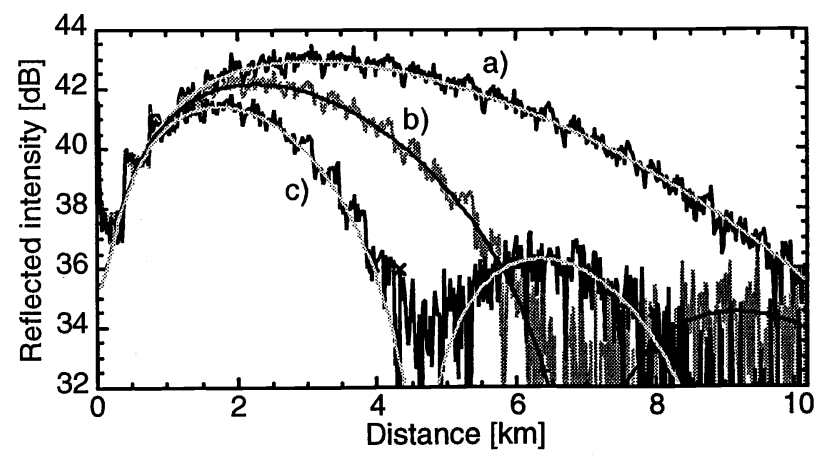

Fig. 3. OTDR traces of the same $765 \mathrm{~m}$ long fiber ring resonator with respective numerical fit of Eq. (7) for rotation rates: a) $0.1 \mathrm{rad} / \mathrm{s}$, b) $0.2 \mathrm{rad} / \mathrm{s}$ and c) $0.3 \mathrm{rad} / \mathrm{s}$.

the step order is getting higher the number of contributions grows but their intensity decays resulting in a maximum of the OTDR trace. It turns out that rotation of the fiber ring also affects the OTDR trace, as shown in Fig. 3. This sensitivity to the Sagnac effect can only be explained considering that some of the different Rayleigh contributions are mutually coherent ${ }^{2}$. Thus the model of the intensity . Rayleigh backscattered signal should consider the Rayleigh backscattered contributions generated in the ring by a coherent probe pulse, that is uniformly scattered while circulating around the fiber ring.

The OTDR trace is made of equal length steps corresponding to the ring length. Each step results from the addition of a definite number of contributions circulating in the ring at the given propagation time. In the referential frame of the ring, the different speeds experienced by the probe pulse and Rayleigh light ( $c_{p}$ and $c_{r}$ respectively) can be expressed according to special relativity:

$$
c_{p}=\frac{c_{0}}{n}-\chi v
$$

$$
c_{r}=\frac{c_{0}}{n}+\chi v
$$

$$
\chi=1-\frac{1}{n^{2}}
$$

where $\chi$ is the Fizeau drag first order correction factor ${ }^{3}, c_{0}$ is the vacuum velocity of light, $n$ is the propagation refractive index through the ring at rest and $v$ is the ring tangential speed ( $v$ is chosen positive in the probe direction).

The electric field resulting from the OTDR probe pulse which made $l$ turns (counterclockwise) in the ring at velocity $c_{p}$, being scattered at a distance $y$ from the coupler and which made $m$ turns back (clockwise) at velocity $c_{r}$ is given by:

$$
E_{l m}(y)=E_{00}(y)(k \xi)^{\frac{l+m}{2}} \exp \left[-\frac{\alpha}{2}(l+m) L\right] \exp \left\{i \beta L\left[(l+m)+\chi v \frac{n}{c_{0}}(l-m)\right]\right\}
$$

where $E_{o o}(y)$ represents the electric field amplitude for $l=m=0$ :

$$
E_{00}(y)=\sqrt{I_{i n} R(y)}(1-k) \xi \exp (-\alpha y) \exp (i 2 \beta y)
$$

where $I_{\text {in }}$ is the input intensity, $\beta$ is the propagation constant, $k$ the intensity coupling coefficient of the coupler, $\xi$ is the intensity transmission coefficient of the ring excluding the fiber linear loss, $R(y)$ is the intensity Rayleigh backscattering coefficient, $\alpha$ is the intensity linear attenuation of the fiber, $L$ is the ring length and $y(0 \leq y \leq L)$ is the distance along the fiber from the coupler to the scattering point.

The ring resonators used for Rayleigh backscattering gyros are preferably several hundred meters long to enhance the Sagnac phase shift due to the ring rotation. It has been formally demonstrated in [1] that such long ring resonators present negligible polarization sensitivity and therefore polarization problems are not taken into account in this description.

The resulting OTDR trace is obtained by summing over $l$ and $m$ each contribution given in Eq. (4) which experienced the same propagation time. As these contributions are generated simultaneously by the same source and also detected simultaneously they must be added coherently. However, as a result of the random character of Rayleigh backscattering, the contributions scattered from different scattering 
centers are not correlated ${ }^{2}$ and must be added incoherently. For a given propagation time, each contribution is scattered only by either of the two different centers spaced by a distance $L / 2$ as described in Fig. 4 for step number 1.

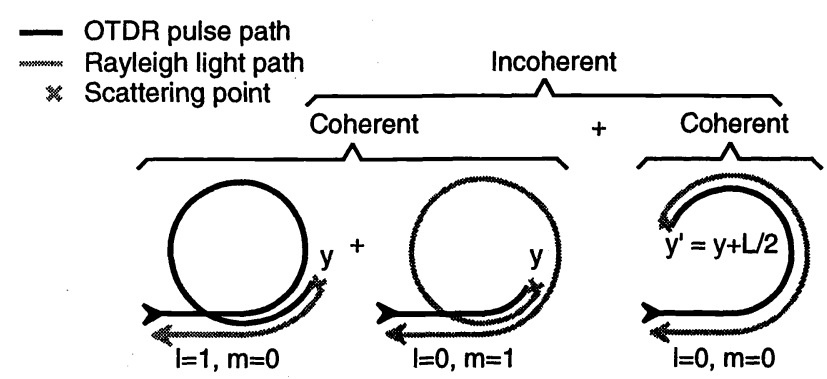

Fig. 4. Addition of the 3 contributions of step 1. The contributions scattered at the same point are added coherently.

These considerations lead to the following discontinuous step-like model of the backscattered intensity signal given by the OTDR:

$$
\begin{aligned}
I_{u}(y)= & I_{o}(y)(k \xi)^{u-1} \exp (-\alpha u L) \\
\times & {\left[k \xi\left|\sum_{p=0}^{\mu} \exp \left[i \beta L \frac{n}{c_{0}} \chi v(2 p-u)\right]\right|^{2}\right.} \\
& \left.+\left|\sum_{p=0}^{u} \overline{1}^{1} \exp \left[i \beta L \frac{n}{c_{0}} \chi v(2 p-u+1)\right]\right|^{2}\right]
\end{aligned}
$$

where $I_{0}(y)=\left|E_{o o}(y)\right|^{2}$ corresponds to the intensity function for step zero, the integer $u=$ int $(z / L)$ is the step order and $z$ is the total propagation distance. The position $y(0 \leq y \leq L / 2)$ of the scattering center is simply related to the total distance by $z=u L+2 y$.

The summations contained in the discontinuous backscattered intensity function [Eq. (6)] can be replaced by the corresponding integral resulting in a continuous function:

$$
\begin{aligned}
I(u)= & I_{\text {in }} R(u) \xi^{2}(1-k)^{2}(k \xi)^{u-1} \exp [-\alpha L(u+1 / 2)] \\
& \times \frac{\left[k \xi \sin ^{2}\left[\beta L \frac{n}{\varepsilon_{0}} \chi v(u+1)\right]+\sin ^{2}\left(\beta L \frac{n}{c_{0}} \chi v u\right)\right]}{\left(\beta L \frac{n}{c_{0}} \chi v\right)^{2}}
\end{aligned}
$$

where $u$ is now a continuous parameter:

$$
u=\frac{z}{L}-\frac{1}{2}
$$

\section{RING LOSS MEASUREMENTS}

When the rotating speed of the fiber ring is null, the backscattered intensity reads:

$$
I(u)=I_{i n} R(u) \xi^{2}(1-k)^{2} \exp \left(-\alpha L\left(u+\frac{1}{2}\right)\right)(k \xi)^{u-1}\left(k \xi(u+1)^{2}+u^{2}\right)
$$

Differentiation of (11) over $z$ followed by a first order approximation yields a simple approximate relation between the position $z_{\max }$ of the maximum backscattered intensity and the resonator parameters (round trip loss: $1-k_{r}$ and coupling coefficient: $k$ ):

$$
k k_{r}=k \xi e^{-\alpha L}=\exp \left(-\frac{2 L}{z_{\max }}\right)
$$

The exponential relationship between $z_{\max }$ and $k_{r}$ results in a highly accurate measurement of the fiber ring loss, especially when the resonator quality factor is high. This makes this simple technique very interesting to characterize fiber ring resonator filters or to build fiber loss sensors based on microbending for instance. Example of additional loss measurement on a $765 \mathrm{~m}$ ring due to fiber bending is given in Fig. 2. Measurement on shorter fiber rings of a few meters ${ }^{1}$ does not make any difference as long as the OTDR is able to resolve the maximum of the trace. 


\section{GYROSCOPIC MEASUREMENTS}

Once the ring parameters $k, L, \alpha, n$ and $\xi$ are known, the rotation rate can be determined using Eq. (7) that is clearly dependent on the ring tangential velocity. At low rotation rates the OTDR trace is only significantly affected after many circulations in the ring (long distances) resulting in an increase of the signal final slope. Fitting Eq. (7) over the OTDR signal is then required. As the rotation rate increases

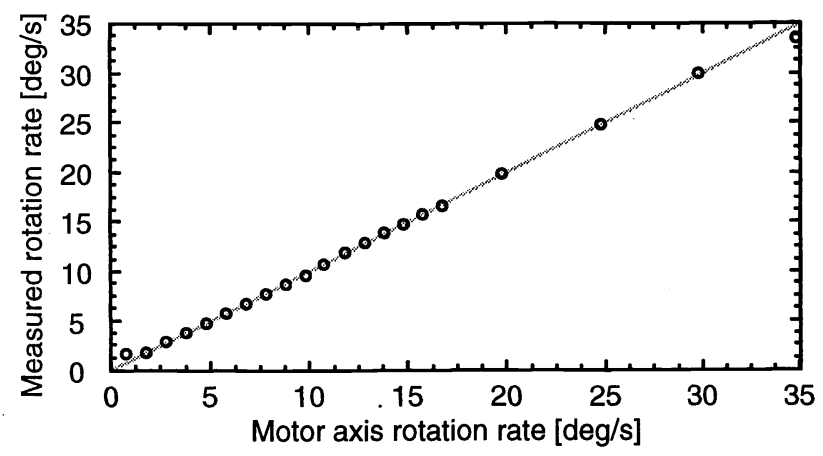

Fig. 5. Measured rotation rate using fitting of expression (8) as a function of the actual rotation rate. the final slope becomes steeper and then other minimas and maximas appear periodically at the end of the OTDR trace (Fig. 3). Position of these minimas are linearly related to the tangential velocity by:

$$
\frac{\lambda c_{0}}{2 L n^{2} \chi}=v
$$

Measurements were performed on a long ring gyroscope made of $765 \mathrm{~m}$ of single-mode fiber spliced to a $5 / 95 \%$ coupler and wounded on a $14 \mathrm{~cm}$ diameter drum. The OTDR traces were obtained with no time averaging from a commercial instrument having a $10 \mathrm{~nm}$ wide spectra at $1.3 \mu \mathrm{m}$. Figure 2 shows the fitting of Eq. (7) on the OTDR traces obtained for different rotation rates. In our experiment this gyroscope was mounted on the axis of a stepping motor. The rotation rate measured by the gyroscope is in excellent agreement with the motor rotation rate, as shown in Fig. 5.

The tangential velocity $v$ and the ring length $L$ are closely related through the ring geometry, so that the Sagnac phase shift responsible for the gyro sensitivity is proportional to the total area enclosed by the fiber loop like in any other fiber-optic gyroscope. It is to notice that Eq. (7) is an even function of $v$ indicating that the gyroscope in this simple configuration is insensitive to the rotation direction. Slow rotation detection and resolution are thus limited by finesse of the fiber ring and the OTDR signal noise that makes two curves measured at very close rotation rates indistinguishable. The obtained resolution of our experimental gyroscope was $0.01 \mathrm{rad} / \mathrm{s}$ without any averaging. For sufficient high rotation rates the signal periodicity and the step length become comparable. The signal is then undersampled by the ring steps and approximation made in Eq. (7) is no longer valid. To overcome this limitation, fitting of the discontinuous relation in Eq. (6) is then required.

\section{CONCLUSION}

Interrogating a fiber ring resonator using an optical time-domain reflectometer (OTDR) is a very simple and highly accurate technique to characterize the finesse of the fiber ring or to monitor the losses experienced by the ring in a sensor. The trace delivered by the OTDR is also sensitive to the gyroscopic motion of the ring making possible to build a low-cost and polarization-insensitive fiber-optic gyroscope with surprisingly good performances. Moreover, the developed model help to understand the unique behavior of Rayleigh backscattering in a fiber ring configuration that can limit Resonant and Brillouin fiber-optic gyroscope sensitivity.

1. A. Küng, J. Budin, L. Thévenaz and P. A. Robert, "Optical fiber ring resonator characterisation by optical timedomain reflectometry", Optics Letters, Vol. 22, No. 2, pp. 90-92, 1997.

2. P. Gysel and R. K. Staubli, "Statistical properties of Rayleigh backscattering in single-mode fiber", J. Light. Tech., Vol. 8, No. 4, pp. 561-567, 1990.

3. H. Lefèvre, "The fiber-optic gyroscope", pp. 11, 1993, Artech House inc., Norwood, MA, USA. 